\title{
Cross-Linking and Immunoprecipitation
}

National Cancer Institute

\section{Source}

National Cancer Institute. Cross-Linking and Immunoprecipitation. NCI Thesaurus. Code C111163.

A type of immunoprecipitation protocol that employs UV-light induced covalent crosslinking of molecules (for instance protein and RNA) prior to the immunoprecipitation and purification steps. 